\title{
Donor-Specific Antibodies in Liver Transplantation
}

\author{
Kathy M. Nilles, M.D., and Josh Levitsky, M.D., M.S.C.I.
}

\section{CASE}

You are concerned about steroid-resistant $\mathrm{T}$ cellmediated rejection (TCMR) in a 39-year-old woman who underwent liver transplantation (LT) for autoimmune hepatitis 2 years ago. Her medical history is notable for three term pregnancies, one of which was complicated by a severe postpartum hemorrhage requiring numerous packed red blood cell transfusions. Her transplant operation also required more than 20 units of blood products intraoperatively. She had two episodes of mild TCMR in the first 6 months, each resolving with augmentation of immunosuppression, and each in the setting of missed doses of immunosuppression resulting in low troughs. She was intolerant to tacrolimus and had been receiving cyclosporine monotherapy, but mycophenolate was added after the first episode of rejection. Her bilirubin and alkaline phosphatase levels are currently normal, but her liver aminotransferase levels have rapidly increased from normal to >200 units/L; biopsy demonstrates "severe acute cellular rejection." She is given intravenous methylprednisolone $500 \mathrm{mg}$ daily for 3 days with prednisone taper and increased cyclosporine, but her alanine aminotransferase level is $627 \mathrm{U} / \mathrm{L}$, aspartate aminotransferase $593 \mathrm{U} / \mathrm{L}$, alkaline phosphatase $422 \mathrm{U} / \mathrm{L}$, bilirubin $13 \mathrm{mg} / \mathrm{dL}$, and platelets $96,000 / \mu \mathrm{L}$. What should be considered next in her evaluation?

\section{INTRODUCTION}

The patient has numerous risk factors (Fig. 1) for formation of donor-specific antibodies (DSAs) with associated antibodymediated rejection (AMR). Her biopsy should be reviewed for histological evidence of AMR. In addition, her serum should be tested for DSAs. If inconclusive, repeat biopsy should be performed to assess either ongoing TCMR or new AMR.

Interest in DSAs and AMR in LT has grown in response to their associations with negative graft outcomes. The following brief review will provide an overview of DSAs in LT for clinicians, including definition, relevance, testing,

Abbreviations: AMR, antibody-mediated rejection; CNI, calcineurin inhibitor; CR, chronic rejection; DSA, donor-specific antibody; HLA, human leukocyte antigen; IVIG, intravenous immunoglobulin; LT, liver transplantation; MELD, Model for End-Stage Liver Disease; MFI, mean fluorescence intensity; PCH, plasma cell hepatitis; PRA, panel-reactive antibody; TCMR, T cell-mediated rejection. From the Department of Medicine and Surgery, Division of Gastroenterology and Hepatology, Comprehensive Transplant Center, Northwestern University Feinberg School of Medicine, Chicago, IL. Potential conflict of interest: Dr. Levitsky owns stock in and consults for Eurofins/Niracor. He is on the speakers' bureau for and recieved grants from Novartis. He is on the speakers' bureau for Gilead.

Received April 19, 2019; accepted August 5, 2019.

View this article online at wileyonlinelibrary.com

(C) 2020 by the American Association for the Study of Liver Diseases 


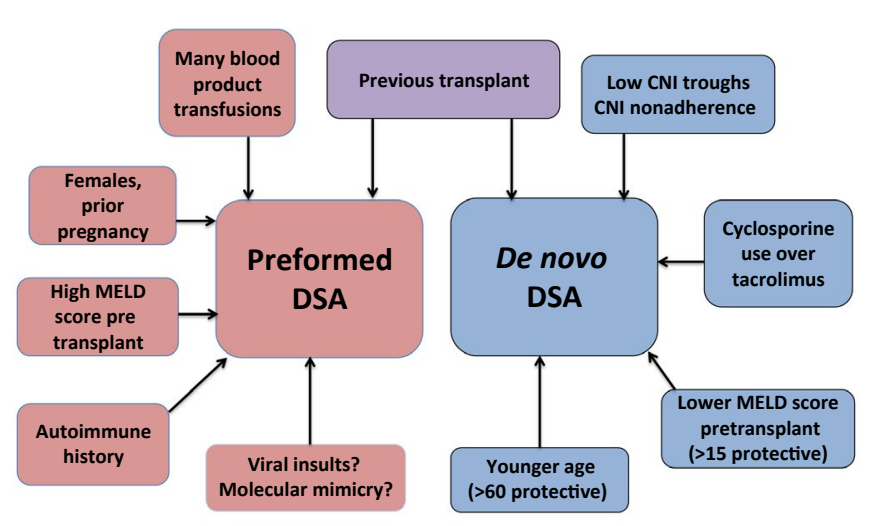

FIG 1 Risk factors for DSA.

and evidence-based management recommendations, with a focus on sentinel studies.

\section{WHAT IS DSA AND WHY IS IT RELEVANT?}

DSAs are antibodies formed by the recipient that can bind to type I and II human leukocyte antigens (HLAs) in the donor organ, resulting in graft injury. Preformed DSAs exist prior to transplant when the recipient has been exposed to a variety of non-self HLAs (Fig. 1), whereas de novo DSAs form after transplantation in response to the new donor organ's HLAs.

Across organ transplantation, DSAs are associated with AMR and graft loss. Unlike other transplants, in LT, DSAs have historically been believed to be clinically irrelevant. Even after positive cross-matches (indicating preformed DSAs) were implicated in increased graft loss, neither DSA testing nor cross-match was routinely performed prior to LT.

However, several cases of acute AMR associated with DSAs in LT emerged and prompted further research. Clinically, acute AMR presents with graft dysfunction or failure, elevated aminotransferase levels, and consumptive thrombocytopenia in the first several weeks after transplant. ${ }^{1}$ This occurs mainly in sensitized patients with preformed DSAs. Chronic AMR generally occurs after formation of de novo DSA. ${ }^{2}$ In both types, histological overlap with TCMR and other forms of allo-immune hepatitis can occur, which lead to formal diagnostic criteria for AMR. ${ }^{3}$ These comprise specific histological vascular injuries involving the hepatic endothelium, c4d staining in the portal/sinusoidal venules, and the presence of serum DSAs, typically of high titer or mean fluorescence intensity (MFI)

Both preformed and de novo DSAs are associated with adverse outcomes in LT (Fig. 2). These include higher risk for overall rejection, including AMR, TCMR, chronic rejection, graft failure, and death. ${ }^{4-10}$ Interestingly, DSAs may have more impact on graft survival in deceased donor than living donor LT, particularly with preformed DSAs. ${ }^{6}$ Finally, DSA has been implicated in preservation injury, plasma cell hepatitis, biliary anastomotic strictures, and graft fibrosis. ${ }^{1,2}$

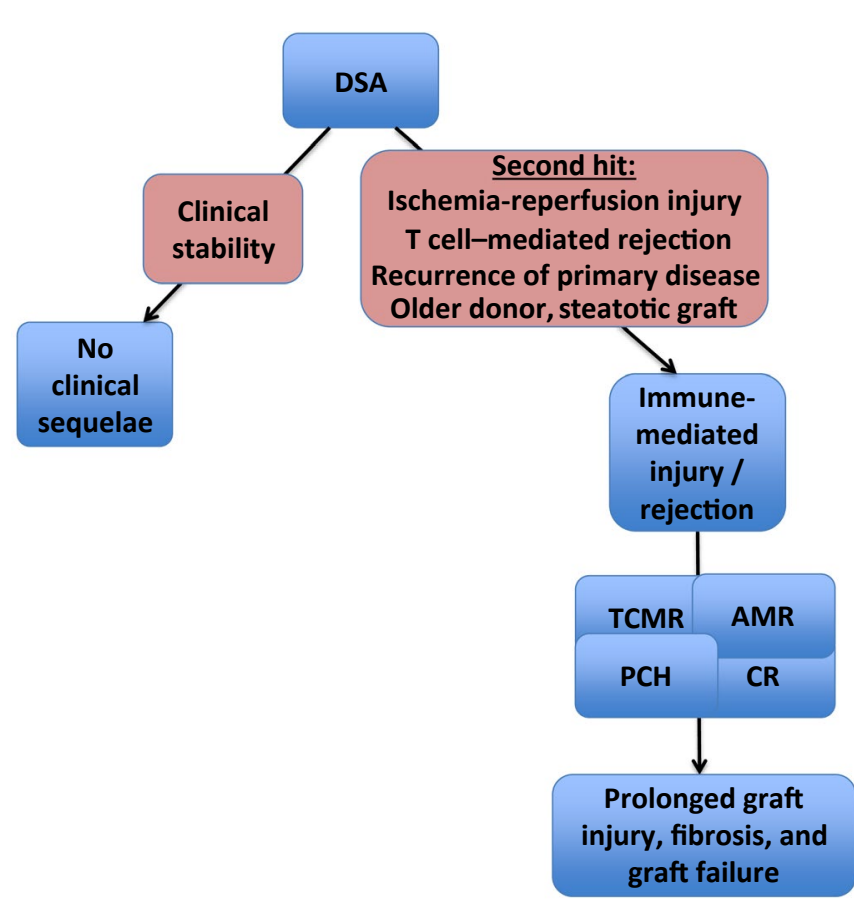

FIG 2 Consequences of DSA.

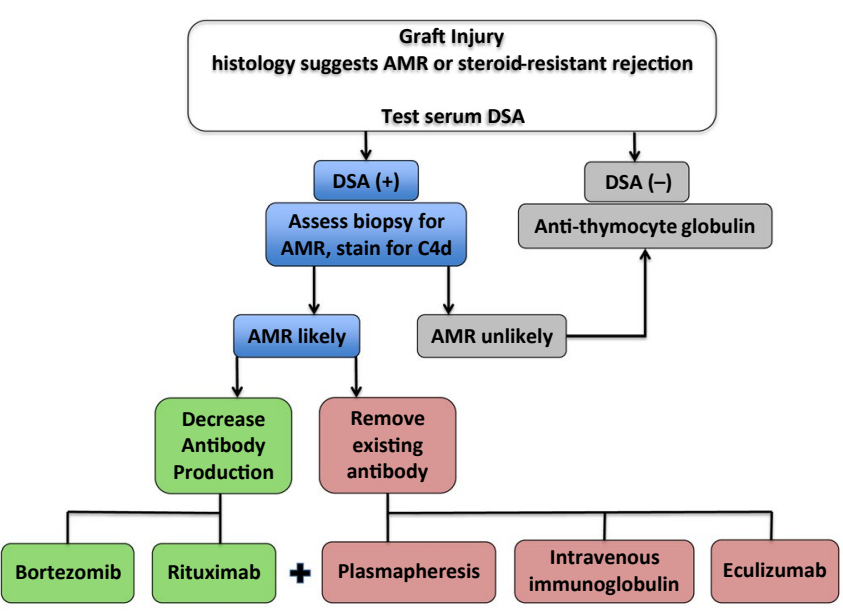

FIG 3 Management of AMR. 
TABLE 1. PREVENTION OF DSA-MEDIATED GRAFT

\section{INJURY}

Pretransplant

- Minimize unnecessary blood product transfusions

- Hemoglobin goals 7-8

- Thromboelastogram to guide transfusions before procedures

- Thrombopoietin receptor agonists over platelets for elective procedures

- Delay elective procedures requiring product transfusion until posttransplant

- Test for DSA in patients with a history of sensitization or high Panel Reactive Antibody

- Consider listing unacceptable antigens*

Peritransplant

- Consider use of induction immunosuppression if high DSA titers

- Consider monitoring of high titers when DSAs are persistent

- Consider pre-emptive therapy if very high titers/MFI of preformed class II DSAs*

Posttransplant

- Use tacrolimus over cyclosporine

- Avoid low trough levels of CNIs

- Timely treatment and resolution of TCMR

- Test for DSA when there is unexplained graft injury

* May be considered controversial.
Most preformed DSAs clear spontaneously early after transplant. ${ }^{4}$ In patients with persistent DSAs (preformed or de novo), a subset develops graft injury, usually in combination with a "second hit" such as preservation injury, TCMR, or poor donor quality. ${ }^{1}$ Preformed class II DSAs, particularly the immunoglobulin G3 subclass, appear to have the highest risk for graft injury and death compared with preformed class I or de novo DSAs. ${ }^{7,8}$ Finally, DSA strength is important; class II DSA with high MFI $(>10,000)$ is associated with the highest risk for rejection. ${ }^{7}$ Identifying which subtypes of DSA are detrimental, and at which levels, are areas of active research.

\section{WHEN SHOULD A CLINICIAN TEST FOR DSA?}

A collaborative effort between the American Society of Transplantation and the American Society for

\section{HLA Donor-Specific Antibody (DSA) Test}

Status: Final result

Linked Results

Procedure Abnormality

Status

Final result

Final result

Final result

DSA Luminex CLII Titers: One Lambda

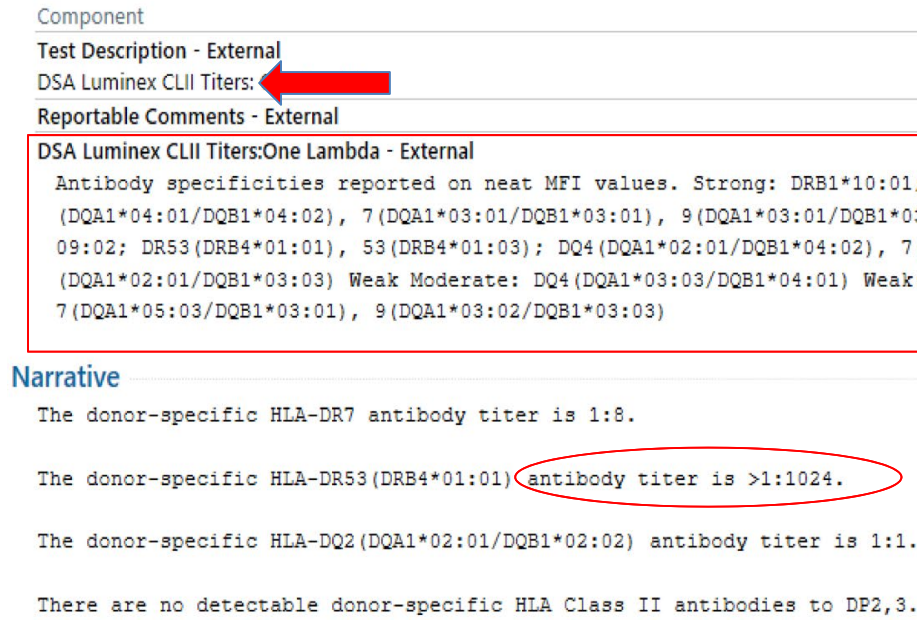

FIG 4 Printout of an actual patient's HLA DSA results. Notably, the subtype of DSA (de novo or preformed) is not distinguished by a laboratory test. Rather this is determined by the date of the serum collection relative to the transplant. DSAs are designated as preformed (prior to transplant if prior exposure to that antigen) or de novo (after transplant) based on the date of the laboratory testing. Arrow: "CLII" denotes that class II DSA is being tested here (this would read "CLI" for testing of class I DSA). Box: denotes all of the HLA antigens detectible in the patient's (recipient's) serum, listed as weak, moderate, and strong. Note that these are not "donor specific" in reference to the donor graft HLA antigens; rather these represent all foreign antigens to which the recipient has formed antibodies. These antigen exposures are obtained from any sensitizing events (such as prior pregnancy or blood transfusions). This is conceptually similar to a PRA. Narrative: this information is specific to the organ donor. This donor is typed as HLA DP2,3, DQ2, DR7, and DR53. This patient (recipient) has antibodies (DSA) to three of the four antigens from the donor. Oval: represents the quantitative DSA titers. The titer of 1:1024 is clinically relevant, whereas the other titers are likely insignificant. Note that in this case, antibody titers, rather than MFI, are reported. 
Histocompatibility and Immunogenetics resulted in publication of the Sensitization in Transplant Assessment Risk, ${ }^{11}$ which may help guide clinicians in DSA testing in LT recipients. Testing for DSA before transplant is strongly recommended in liver patients who need dual organ transplant. For LT alone, recommendations are based on expert opinion. We propose DSA testing for two purposes. First, pretransplant DSAs can predict rejection risk, because sensitized patients with high panel-reactive antibodies (PRAs) have higher risk for immunological graft injury. Knowledge of pretransplant DSAs is also helpful when graft injury is prolonged or unexplained. The second purpose of DSA testing is posttransplant, in settings of severe or steroidrefractory TCMR or biopsy suggesting $A M R{ }^{3}$ Repeat DSA testing is recommended when AMR is treated to follow treatment response and risk-stratify for future events.

\section{HOW AND WHEN TO TREAT DSA AND AMR?}

Given the paucity of published data, management of AMR in LT recipients is adapted from nonhepatic (predominantly kidney) transplantation. Initial approaches aim to block, inactivate, and remove active circulating antibodies, followed by strategies to decrease current and future antibody production (Fig. 3). Combinations of steroid pulses, intravenous immunoglobulin (IVIG), plasmapheresis, rituximab, and anti-thymocyte globulin have been used. ${ }^{1,2}$ The proteasome inhibitor bortezomib depletes plasma cells and has been effective in LT recipients. ${ }^{1}$ In addition, the C5 complement inhibitor eculizumab inactivates antibodies and may be considered in refractory AMR.

Treatment risks of over-immunosuppression (opportunistic infections, lymphoma, other malignancies) must be considered. Isolated DSA without evidence of histological graft injury and graft antibody deposition (C4d) generally is not treated. Table 1 outlines methods to prevent DSA formation, which may include reducing sensitization, induction, and tacrolimus-based immunosuppression. ${ }^{1}$

\section{CASE RESOLUTION}

A repeat biopsy showed improving TCMR but evidence of AMR with endothelial injury and sinusoidal C4D staining. Serum DSA titer was 1:1024 (Fig. 4) and MFI was 30,000. She was treated with plasmapheresis, IVIG, and bortezomib with preservation of graft function.

\section{CONCLUSION}

DSAs, in a subset of LT recipients, can lead to AMR, graft loss, and decreased survival. DSA testing may help predict rejection risk in sensitized patients. Patients with refractory TCMR or features of AMR on biopsy should have DSA testing. Treatment includes removing circulating DSAs and preventing DSA formation, but additional research is needed in optimal monitoring and management.

\section{CORRESPONDENCE}

Josh Levitsky, M.D., M.S.C.I., Department of Medicine and Surgery, Division of Gastroenterology and Hepatology, Comprehensive Transplant Center, Northwestern University Feinberg School of Medicine, Chicago, IL 60601. E-mail: josh.levitsky@nm.org

\section{REFERENCES}

1) Kim PT, Demetris AJ, O'Leary JG. Prevention and treatment of liver allograft antibody-mediated rejection and the role of the 'two-hit hypothesis'. Curr Opin Organ Transplant 2016;21:209-218.

2) Del Bello A, Congy-Jolivet N, Danjoux $M$, et al. Donor-specific antibodies and liver transplantation. Hum Immunol 2016;77:1063-1070.

3) Demetris AJ, Bellamy C, Hubscher SG, et al. 2016 Comprehensive update of the Banff Working Group on Liver Allograft Pathology: introduction of antibody-mediated rejection. Am J Transplant 2016; $16: 2816-2835$

4) Kozlowski $T$, Rubinas $T$, Nickeleit $V$, et al. Liver allograft antibodymediated rejection with demonstration of sinusoidal C4d staining and circulating donor-specific antibodies. Liver Transpl 2011;17:357-368.

5) Musat Al, Agni RM, Wai PY, et al. The significance of donor-specific HLA antibodies in rejection and ductopenia development in $A B O$ compatible liver transplantation. Am J Transplant 2011;11:500-510.

6) Levitsky J, Kaneku H, Jie C, et al. Donor-specific HLA antibodies in living versus deceased donor liver transplant recipients. Am J Transplant 2016;16:2437-2444

7) O'Leary JG, Kaneku H, Jennings LW, et al. Preformed class II donorspecific antibodies are associated with an increased risk of early rejection after liver transplantation. Liver Transpl 2013;19:973-980.

8) O'Leary JG, Kaneku H, Banuelos N, et al. Impact of IgG3 subclass and C1q-fixing donor-specific HLA alloantibodies on rejection and survival in liver transplantation. Am J Transplant 2015;15:1003-1013.

9) O'Leary JG, Kaneku H, Susskind BM, et al. High mean fluorescence intensity donor-specific anti-HLA antibodies associated with chronic rejection postliver transplant. Am J Transplant 2011;11:1868-1876.

10) Kaneku H, O'Leary JG, Banuelos N, et al. De novo donor-specific HLA antibodies decrease patient and graft survival in liver transplant recipients. Am J Transplant 2013;13:1541-1548.

11) Tambur AR, Campbell $P$, Claas $F H$, et al. Sensitization in Transplantation: Assessment of Risk (STAR) 2017 Working Group Meeting Report. Am J Transplant 2018;18:1604-1614. 